\title{
Metal Stenting with or without Endobiliary Radiofrequency Ablation for Unresectable Extrahepatic Cholangiocarcinoma
}

\author{
Huihong Liang1, Zhenwei Peng², Liangqi Cao', Shikun Qian1, Zili Shao1* \\ ${ }^{1}$ Department of Hepatobiliary Surgery, The Second Affiliated Hospital of Guangzhou Medical University, \\ Guangzhou, China \\ ${ }^{2}$ Department of Oncology, The First Affiliated Hospital of Sun Yat-sen University, Guangzhou, China \\ Email: zilishao@163.com
}

Received 31 August 2015; accepted 13 October 2015; published 16 October 2015

Copyright (C) 2015 by authors and Scientific Research Publishing Inc.

This work is licensed under the Creative Commons Attribution International License (CC BY).

http://creativecommons.org/licenses/by/4.0/

(c) ()

\section{Abstract}

Background: Retrospective comparison of stent patency and survival of patients with unresectable extrahepatic cholangiocarcinoma (CCA) treated by placement of self-expanding metal stents (SEMS) with or without endobiliary radiofrequency ablation (ERFA). Methods: Between January 2010 and September 2014, 76 patients with biliary obstruction caused by unresectable extrahepatic CCA (27 patients with Bismuth type I hilar CCA and 49 patients with distal CCA) were recruited into this study. Patients either received ERFA with SEMS $(n=34)$ or SEMS only $(n=42)$. Stent patency and survival of both groups were compared and predictors for overall survival were analyzed. Results: Demographic data were not different between the two groups. Complication rates of both groups were similar. Thirty-day stent patency was obtained in all patients and the median stent patency in the ERFA + SEMS group (9.5 months, 4.5 - 14.0 months) was significantly longer than that in the SEMS group (8.4 months, 4.9 - 11 months $)(P=0.024)$. The overall survival curve in the ERFA + SEMS group was significantly better than that of the SEMS group $(P=0.036)$. Multivariate Cox proportional hazards regression analyses showed that ERFA, tumor AJCC stage, extrahepatic CCA type, serum albumin and adjuvant chemotherapy were significant prognostic factors for overall patient survival. Conclusions: ERFA is effective for unresectable extrahepatic CCA and may improve metal stent patency and patient survival for unresectable extrahepatic CCA with biliary obstruction. Prospective randomized trials will be needed to confirm these findings.

\section{Keywords}

Cholangiocarcinoma, Endoscopic Retrograde Cholangiopancreatography, Percutaneous

\footnotetext{
"Corresponding author.
}

How to cite this paper: Liang, H.H., Peng, Z.W., Cao, L.Q., Qian, S.K. and Shao, Z.L. (2015) Metal Stenting with or without Endobiliary Radiofrequency Ablation for Unresectable Extrahepatic Cholangiocarcinoma. Journal of Cancer Therapy, 6, 981992. http://dx.doi.org/10.4236/jct.2015.611106 


\section{Introduction}

Extrahepatic cholangiocarcinoma (EH-CCA) was reported with the highest incidence in eastern and southeastern Asia. It is linked to liver fluke infestations. EH-CCA is a very aggressive tumor and remains highly resistant to current chemoradiation therapies. Radical resection offers the best outcome [1]-[3] but, despite significant advances in diagnostic modalities, the vast majority of patients present with metastases or with advanced locoregional disease that prevents surgical therapy [2]. Even for patients who undergo surgical treatment, recurrent disease is common. Although the tumor burden is usually lethal, the vast majority of patients with EH-CCA present with malignant biliary obstruction, which leads to cholangitis and liver failure, which is also a cause of death. For this reason, the relief of biliary obstruction is a key palliative treatment option for patients with hilar or distal EH-CCA.

Biliary drainage affected by self-expandable metallic stents (SEMSs), is a desirable method of palliation of malignant biliary obstruction for patients whose life expectancy is greater than 3 months [3]. This process has a median stent patency lasting 6 - 10 months [4]-[8]. The major drawback of this treatment is that the patency of the stent is limited to a median duration of 6 - 8 months in up to $50 \%$ of patients. This is mainly due to tumor inor over-growth, biofilm deposition, biliary sludge, or formation of granulation tissue, all of which may result in ongoing or recurrent biliary obstruction that causes significant morbidity and mortality [9].

Several methods, such as the use of organic polymers to coat self-expandable metallic stents, substituting alloys like nitinol for stainless steel, or endobiliary photodynamic therapy (PDT), have been proposed to increase the duration of stent patency in malignant biliary obstruction but they have not been proven to be effective by sufficient data [8] [10]-[12]. Rather, they present with increased risks of side effects, such as pancreatitis and cholecystitis due to covered SEMS and cholangitis and photosensitivity due to PDT [8] [11] [12].

Recently, endobiliary radiofrequency ablation (ERFA) has emerged as a new treatment modality to improve SEMS patency and has been used for unresectable malignant biliary obstruction [13]-[19]. The current work highlights two major advantages of RFA. First, it reduces the tumor load, and thus, delays tumor growth, both of which may prolong stent patency. Second, endobiliary RFA may be suitable for development into a neoadjuvant therapy in unresectable CCA.

Here, the effects of SEMS placement with and without ERFA on stent patency and patient survival for unresectable extrahepatic CCA patients were compared. In this study, qualified patients with EH-CCA were included, and they were treated by stenting alone or in combination after endobiliary RFA. The outcomes (overall survival) of the patients were studied, and multivariate Cox proportional hazards regression analyses were used to identify other significant prognostic factors relevant to overall patient survival.

\section{Methods}

\subsection{Patients Selection}

This retrospective study was approved by the institutional review board of the second affiliated hospital of Guangzhou Medical University. Between January 2010 and September 2014, 242 patients with extrahepatic CCA were treated in our hospital. Seventy-six patients were included in this study using the following criteria and the detailed selection process is described in Figure 1.

1) Inclusion criteria: a) Patients with histologically or cytologically confirmed extrahepatic biliary adenocarcinoma but ineligible for curative surgery due to locally advanced or metastatic disease or unfit for/not willing to undergo a major operation; b) patients who successfully underwent SEMS placement with or without ERFA as initial treatment for obstructive jaundice by endoscopic retrograde cholangiopancreatography (ERCP) or percutaneous transhepatic cholangiography (PTC) approaches if ERCP fails.

2) Exclusion criteria: a) Patients with obstructive jaundice caused by other malignant tumors (tumors of the ampulla of Vater, pancreatic tumors, hepatocellular carcinoma with endobiliary tumor thrombus, bile duct compressed by hilar lymph metastases, e.g.), or patients who had developed obstructive jaundice due to recurrent tumors after initial resection of the CCA; b) patients with Bismuth type II, III, or IV hilar CCA [21] [22]; 


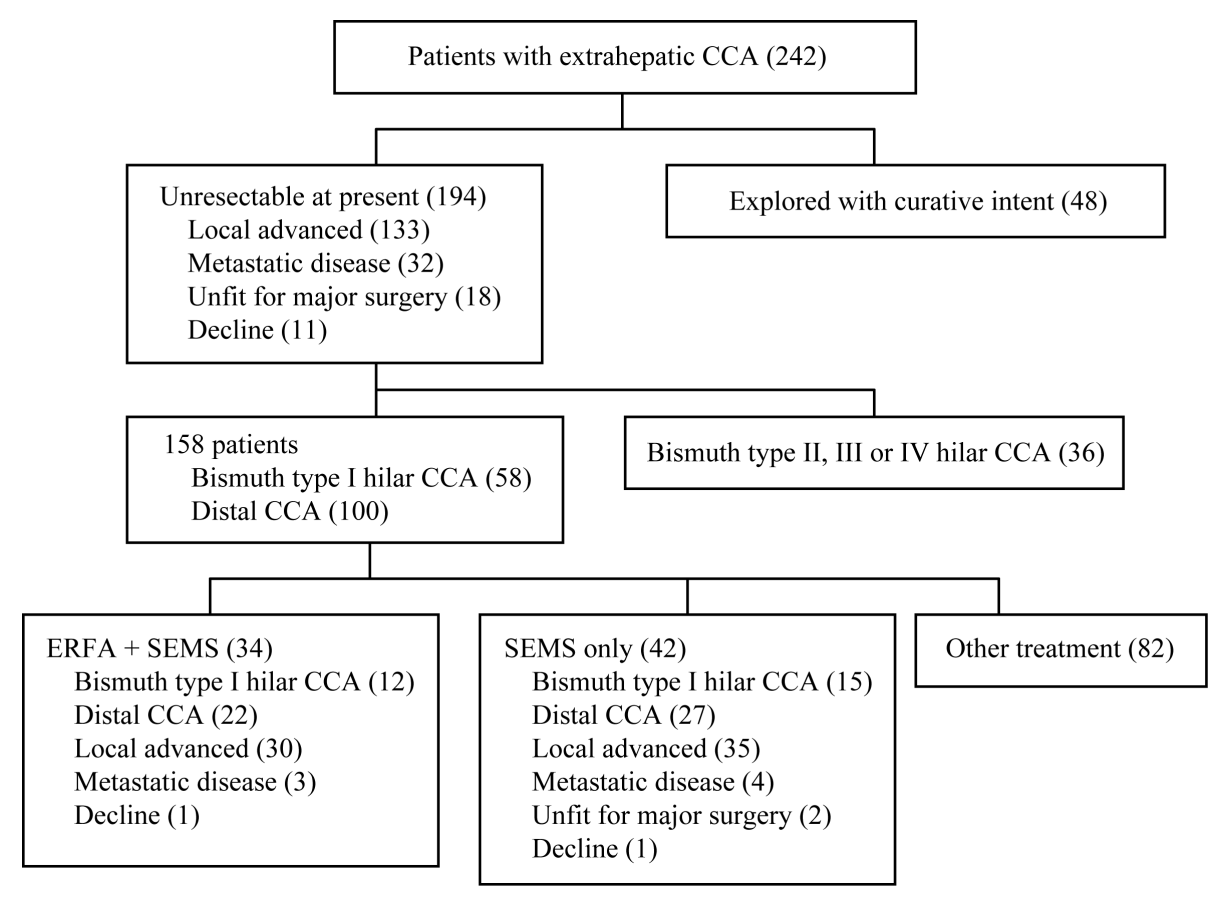

Figure 1. Flow diagram of patient selection process. CCA, cholangiocarcinoma; ERFA, endobiliary radiofrequency ablation; SEMS, self-expanding metal stent.

c) patients who underwent unsuccessful SEMS placement with or without ERFA, who underwent other local ablative treatments (PDT) instead of ERFA, or who underwent placement of plastic stents instead of SEMS for obstructive jaundice.

\subsection{Patient Evaluation}

Pre-treatment assessment of all patients included a complete history and physical examination for general health, laboratory tests and radiologic imaging. A dual-phase contrast-enhanced spiral computed tomography and/or magnetic resonance cholangiopancreatography were performed out on all patients to evaluate biliary tumors and possible vascular involvement. If needed, positron emission tomography with [(18)F]fluoro-2-deoxy-D-glucose (FDG-PET) was used to identify occult metastatic disease. All data were reviewed by a multidisciplinary treatment team of radiologists, surgeons, pathologist and oncologists to select treatment options. For those with unresectable extrahepatic CCA, the option of ERFA before SEMS placement and its potential benefits for prolonging stent patency were discussed with patients, who would make the ultimate decision. Informed consent was obtained from all patients.

\subsection{ERFA and/or SEMS Treatment and Follow-Up}

All patients received intratracheal general anesthesia. Side viewing endoscopes TJF-160 (Olympus America, Center Valley, PA) were used for all endoscopical procedures. Patients underwent biliary sphincterotomy followed by cholangiogram to confirm stricture location. An endoscopical clamp or cytology brush was use to obtain biopsies for histological analysis. Percutaneous biopsy and cholangiography were performed under real-time US guidance (EUB-2000, HITACHI Medical Systems) for all percutaneous approaches.

For patients who received ERFA, the Habib EndoHPB catheter (EMcision UK, London, United Kingdom) was advanced to the stricture location, and radiofrequency energy was delivered by an RFA generator (1500 RF generator; RITA Medical Systems Inc, Fremont, CA) at $400 \mathrm{kHz}$ at $10 \mathrm{~W}$ for $2 \mathrm{~min}$, with a rest period of one min before moving the catheter. Repeat energy was applied to ensure the whole stricture length was covered. An uncovered (Cook Ireland ltd, Limerick, Ireland) or covered SEMS (WallFlex RX RMV, Boston Scientific Corporation, city, MA) was placed after ERFA (Figure 2).

All patients received computed tomography and/or MRI and laboratory blood tests about 30 days after the 


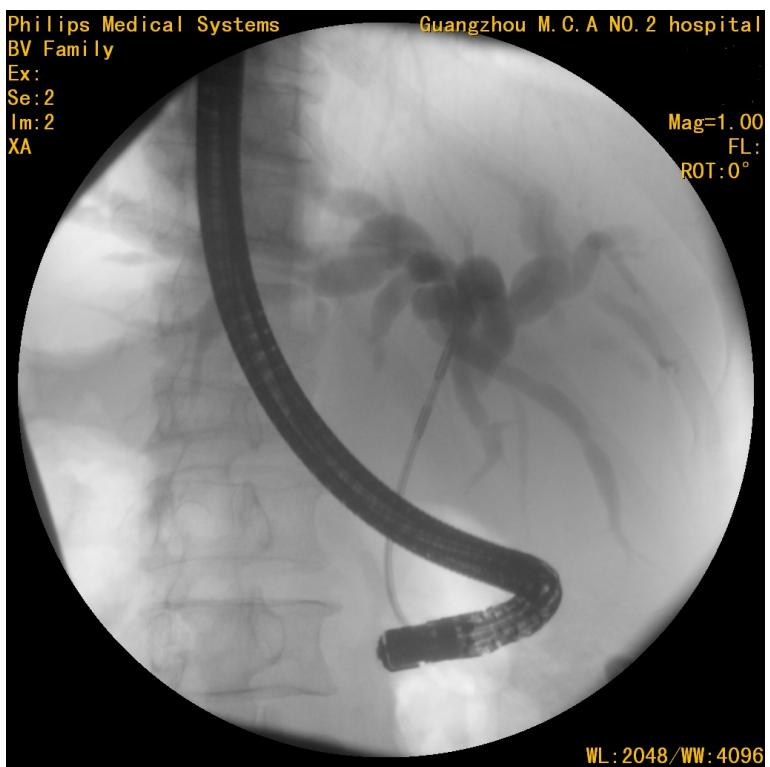

(a)

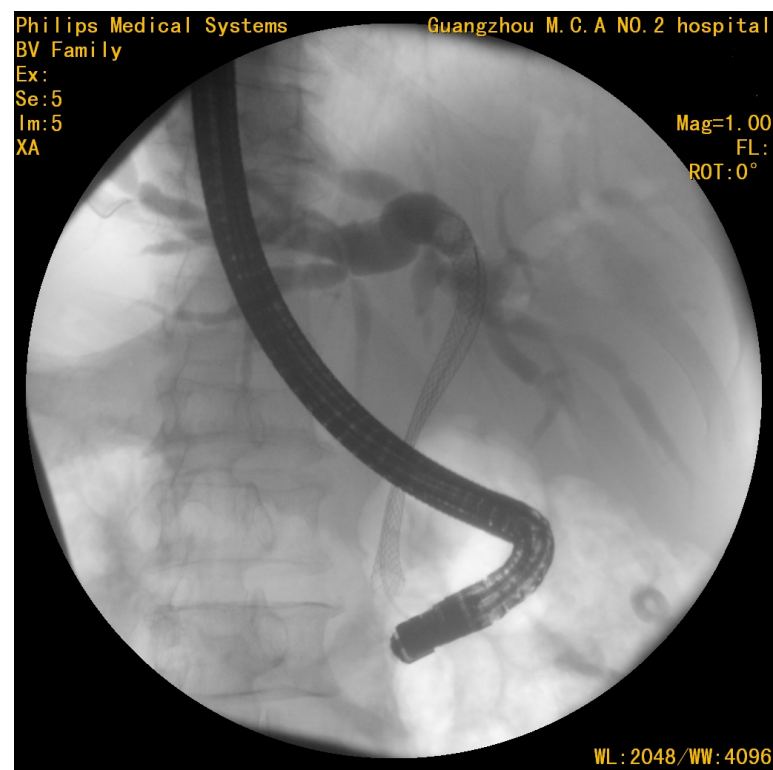

(b)

Figure 2. Fluoroscopic imaging of the procedure of ERFA and stent placement. (a) This is a 46 year old male with unresectable Bismuth type I hilar CCA. Habib EndoHPB catheter was deployed to ablate bile duct tumor approached by endoscopic retrograde cholangiopancreatography; (b) after completing ERFA, an uncovered self-expanding metal stent was placed at the bile duct stricture site.

procedure and bile duct stricture improvement was evaluated. Those with obvious bile duct decompression and persistent liver function improvement (total bilirubin and combined bilirubin decreasing) were considered to have full stricture improvement. Partial or no improvement was identified when radiologic images showed that a stricture remained in the previous tumor site and bilirubin did not normalize or bilirubin increased after decreased.

Adjuvant chemotherapy based on practice guidelines of the National Comprehension Cancer Network (NCCN) in hepatobiliary cancers was recommended for patients who recovered from the intervention [20]. All patients were regularly followed up every two months since the last intervention procedure until November 2014. When bile ducts occluded again, the multidisciplinary treatment team met to decide whether a repeat ERFA and/or SEMS placement or percutaneous biliary drainage was appropriate. Major complications were defined as procedure-related complications resulting in an unplanned increase in medical care, prolonged hospitalization, permanent adverse sequelae, or death [21].

\subsection{Statistical Analysis}

Demographic data for both groups was compared using the Student's $t$ test for continuous data and the Chi-squared test for categorical data. The median stent patency was compared by Mann-Whitney test. Overall survival curves from the date of ERFA and/or SEMS were calculated and constructed by the Kaplan-Meier method and compared using the log-rank test. The relative prognostic significance of the variables in predicting overall survival was assessed by multivariate Cox proportional hazards regression analysis. Statistical analyses were performed using SPSS 10.0 statistical software (SPSS Company, Chicago, IL). Continuous data are given as means \pm S.D. All statistical tests were two-sided, and a significant difference was considered when $\mathrm{P}<0.05$.

\section{Results}

\subsection{Patient Groups}

Seventy-six patients with indication for SEMS and ERFA were included in this study, among which, 34 patients opted to receive ERFA followed by SEMS placement and 42 patients opted to receive SEMS only. There were no significant differences among the patients in demography (gender, age), tumor grade, and staging, patients' 
general health conditions, and liver function parameters (Table 1). Most patients (72/76, 94.7\%) had unresectable extrahepatic CCA due to local advanced tumors $(n=65)$ or metastatic disease $(n=7)$. Two patients with potentially resectable extrahepatic CCA refused tumor resection, and 2 patients were considered unfit for a major operation (one had severe cirrhosis; the other had poor pulmonary function). Histological and/or cytological diagnosis of biliary adenocarcinoma was made in 66 patients by ERCP. For those who underwent a PTC approach, 6 patients also had hepatic metastatic tumors and the other 4 patients had Bismuth type I hilar CCA. Percutaneous biopsy was successfully performed on hepatic metastatic tumors or hilar CCA. There was no difference in

Table 1. General clinical data of the patients.

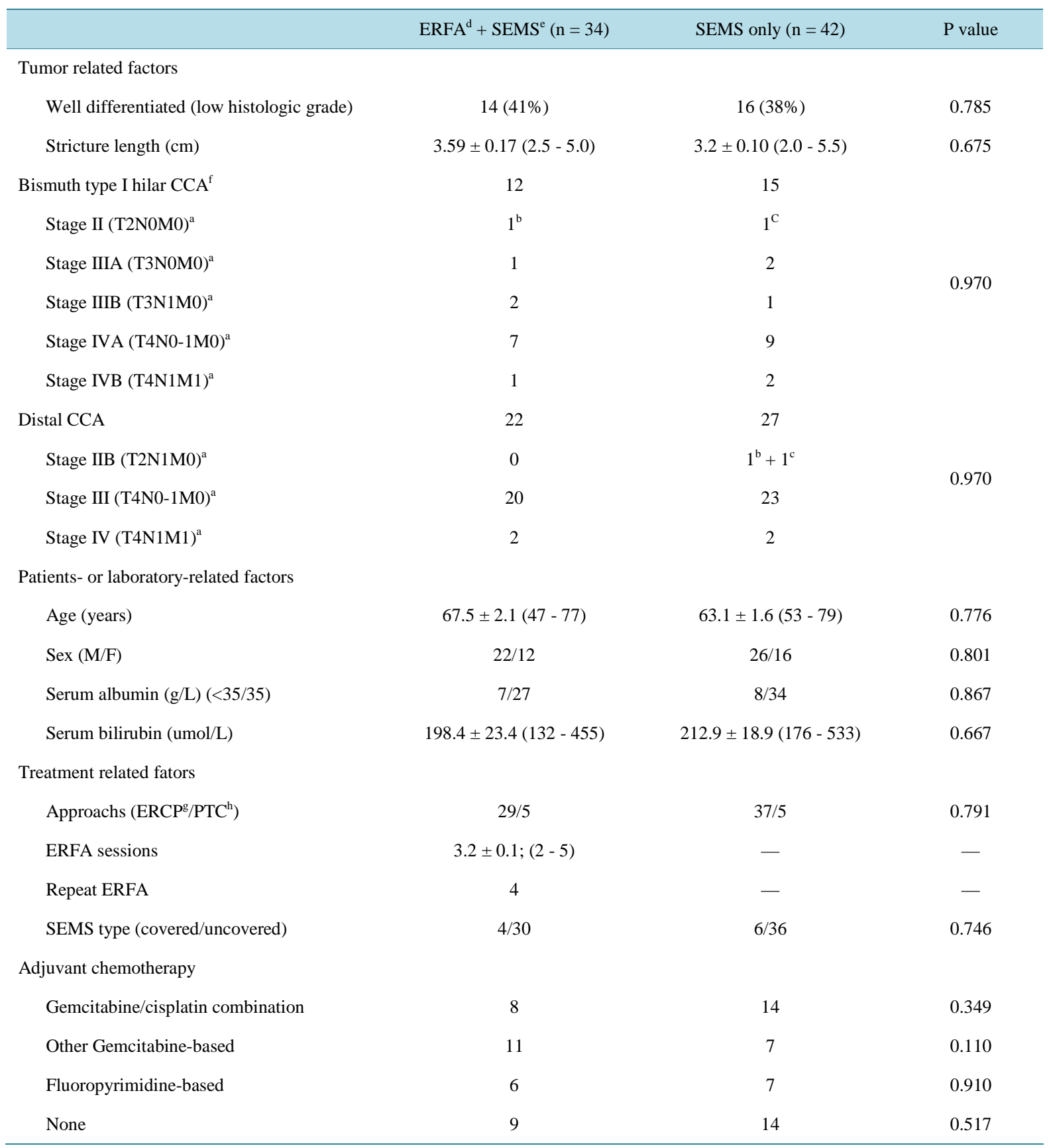

${ }^{\mathrm{a}}$ American Joint Committee on Cancer (AJCC) TNM Staging (7th edition 2010). 27; ${ }^{\mathrm{b}}$ Patients who declined to have major operation; ${ }^{\mathrm{C}}$ Patients who were unfit for major operation; ${ }^{d}$ Endobiliary radiofrequency ablation; ${ }^{\text {e}}$ Self-expanding metal stent; ${ }^{\mathrm{f}}$ Cholangiocarcinoma; ${ }^{\mathrm{g}}$ Endoscopic retrograde cholangiopancreatography; ${ }^{\mathrm{h}}$ Percutaneous transhepatic cholangiography. 
follow-up duration between the two groups (ERFA + SEMS: 12.2 months \pm 0.53 , range, 6 - 19.5 months; SEMS only: 11.3 months \pm 0.34 , range, 7 - 16 months) (t-test, $\mathrm{P}=0.078$ ).

\subsection{Treatment Efficiency and Complications}

Thirty-day stent patency was obtained in all patients and the median stent patency in ERFA + SEMS group (9.5 months, range: 4.5 - 14.0 months) was significantly longer than that in the SEMS group (8.35 months, range: 4.9 11 months) (Mann-Whitney test, $\mathrm{P}=0.024$ ). During follow up, 4 patients in the ERFA + SEMS group received repeated ERFA, but only 2 patients completed the process proposed by Steel and co-workers, which required an ablation of 2 min at 10W [17]. The other two patients failed due to technical issues: a short-circuit produced in the primary stents during the early process, which led to the termination of the repeated ERFA. Four patients received a secondary SEMS placement within the primary stent successfully. Sixty-one patients received percutaneous biliary drainage when biliary obstruction relapsed during the followup. Twenty-three patients refused to have adjuvant therapy either due to costs or anxiety about adverse effects.

There was no treatment procedure-related mortality in the study subjects and no significant difference in treatment complications was observed between the two groups (Table 2). Twelve patients had moderate or severe post-procedure abdominal pain, which required oral or intravenous analgesics for relief of symptoms. Three patients developed cholangitis and one had pyogenic cholecystitis which required percutaneous transhepatic gallbladder drainage. All of these patients recovered fully after antibiotic treatment. Three patients were diagnosed with asymptomatic pancreatitis (serum amylase > $1000 \mathrm{IU} / \mathrm{L}$ ) and recovered after conservative treatment.

\subsection{Survival and Prognosis Analysis}

Nine patients survived to the end of the follow-up period in November 2014. Of the patients who died, a detailed list of causes of death is summarized in Table 3. The major causes of death were disease progression (liver failure), bleeding, biliary infection, cachexia, and lung infection. The rates of occurrence of each of these showed no significant differences between the two both groups. None of the patients died from ERFA- or SEMS-related procedures.

Kaplan-Meier plots for ERFA + SEMS and SEMS groups associated with patient survival are shown in Figure 3. The log-rank test demonstrated that the overall survival in the ERFA + SEMS group $(n=34)$ was significantly longer than that in the SEMS group $(\mathrm{n}=42)(\mathrm{P}=0.036)$, as shown in Figure 3(a). Further subgroup analysis excluded patients with metastatic disease $(n=7)$, or unfit $(n=2)$ or declined surgery $(n=2)$. The

\begin{tabular}{ccc}
\hline Table 2. Complications after treatments. & \\
\hline Complications & ERFA $^{\mathrm{a}}+\mathrm{SEMS}^{\mathrm{b}}(\mathrm{n}=9)$ & SEMS only $(\mathrm{n}=10)$ \\
\hline Abdominal pain & 5 & 7 \\
cholangitis & 1 & 2 \\
Pyogenic cholecystitis & 1 & 0 \\
Asymptomatic pancreatitis & 2 & 1 \\
\hline
\end{tabular}

${ }^{\mathrm{a}}$ Endobiliary radiofrequency ablation; 'Self-expanding metal stent.

\begin{tabular}{ccc|}
\hline \multicolumn{2}{l}{ Table 3. Causes of death after treatment. } \\
\hline Causes of deaths & ERFA $^{\mathrm{a}}+$ SEMS $^{\mathrm{b}}(\mathrm{n}=31)$ & SEMS only $(\mathrm{n}=36)$ \\
\hline Liver failure & 23 & 27 \\
Bleeding & 2 & 4 \\
Biliary infection & 3 & 2 \\
Cachexia & 2 & 3 \\
Lung infection & 1 & 0 \\
\hline
\end{tabular}

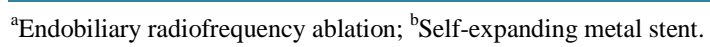




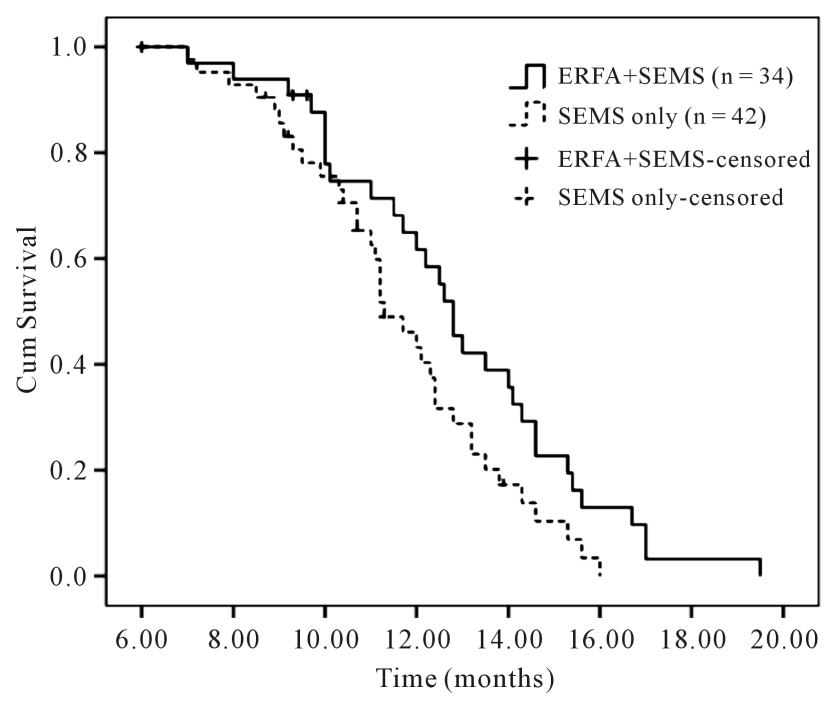

(a)

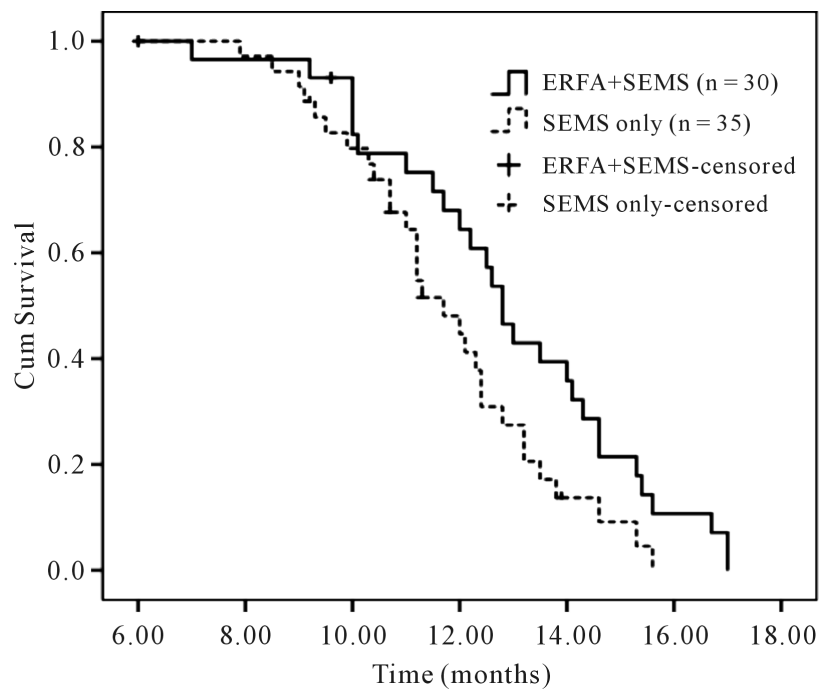

(b)

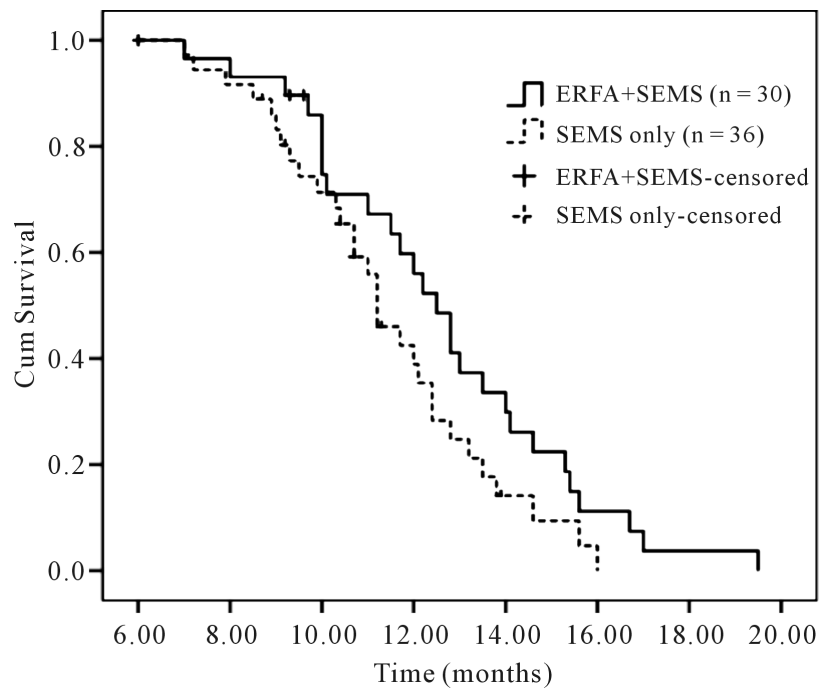

(c) 


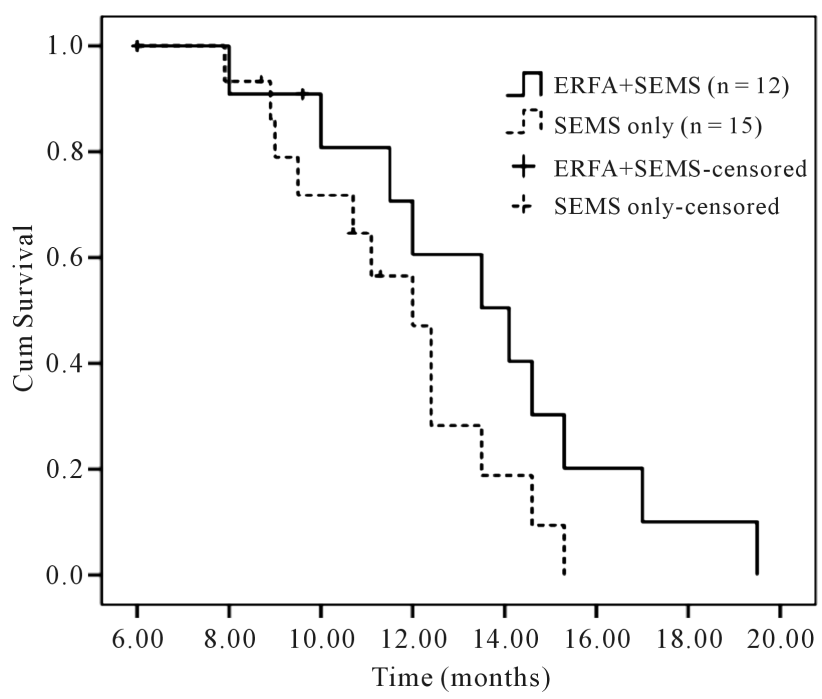

(d)



(e)

Figure 3. Kaplan Meier Survival Analysis for the Patient Cohorts. (a) Overall survival curve for patients with unresectable extrahepatic cholangiocarcinoma treated with ERFA followed by placement of SEMS or SEMS alone. The results demonstrate that ERFA + SEMS is better than that of SEMS placement alone (log-rank test, $\mathrm{P}=0.036$ ). (b) Overall survival curve for patients with locally advanced unresectable extrahepatic CCA (those patients with metastatic disease, or unfit for major surgery, or declined to have surgery were excluded) treated with endobiliary radiofrequency ablation (ERFA) followed by placement of self-expanding metal stent (SEMS) or SEMS placement alone. The graph shows the ERFA + SEMS is better than that of SEMS placement alone (log-rank test, $\mathrm{P}=0.034)$. (c) Overall survival curves for patients with unresectable extrahepatic CCA treated with uncovered SEMS with/without ERFA (those with cover metal stent placement were excluded) (log-rank test, P $=0.084$ ). (d) Overall survival curves for patients with unresectable Bismuth type I hilar CCA (those with distal cholangiocarcinoma were excluded) treated with SEMS with/without ERFA (log-rank test, $\mathrm{P}=0.116$ ). (e) Overall survival curves for patients with unresectable distal CCA (those with hilar cholangiocarcinoma were excluded) treated with SEMS with/without ERFA (log-rank test, $\mathrm{P}=0.214$ ). 
log-rank test demonstrated that the overall survival for patients with local advanced extrahepatic CCA in the ERFA + SEMS group $(n=30)$ was significantly longer than in the SEMS group $(n=35)(P=0.034)$, as shown in Figure 3(b). If patients with covered metal stents $(n=10)$ were excluded from the study, the overall survival in the ERFA + SEMS group was longer than in the SEMS group, but the difference was not significant (log-rank test, $\mathrm{P}=0.084$ ) as in Figure 3(c). Similarly, no significant differences were found between the two treatments with respect to unresectable Bismuth type I hilar CCA (log-rank test, P = 0.116) as in Figure 3(d); or unresectable distal CCA (log-rank test, $\mathrm{P}=0.214$ ) as in Figure 3(e).

Multivariate Cox proportional hazards regression analyses showed that ERFA, tumor AJCC stage, extrahepatic CCA type, serum albumin and adjuvant chemotherapy were prognostic factors for overall patient survival (Table 4).

\section{Discussion}

ERFA was first introduced by Steel and co-workers in 2011 as a palliative treatment modality for malignant obstructive jaundice [17]. Since then, several studies have reported the outcomes of ERFA in the treatment of malignant biliary obstruction [13]-[16] [18] [19]. In these studies, the safety of ERFA was confirmed. As in those cases, very few complications were here found to be associated with endobiliary RFA combined with SEMS, and no procedure-related mortality was observed. Cholangitis was observed in only one case and asymptomatic pancreatitis in 2 cases in the ERFA + SEMS group, but all of them fully recovered after conservative treatment. No bile duct perforation or vessel thrombus was observed. Since the introduction of the ERFA catheter in 2011, thermal injury to adjacent structures around the bile duct was a main concern [14] [17]. However, such concern seems to be unnecessary. Unlike traditional RFA electrodes for solid tumors, the bipolar Habib EndoHPB RFA catheter seems confined to ablation of small necrotic zones. Even in ex-vivo pig livers, this catheter could only ablate a semidiameter of $4.5 \mathrm{~mm}$ under energy output of $10 \mathrm{~W}$ in $60-90 \mathrm{~s}$ [13]. This may be much more confined in vivo when flow of blood of the adjacent structure serve as cooling stream which could take away much heat based in the experience in ablation of solid tumors [22]. RFA is in-situ ablative technique and tissue structure remains structurally intact immediately after RFA. Furthermore, SEMS placement leads to immediate decompression within the bile duct, which could prevent bile duct perforation. The vessel around the ablation could be preserved against thrombus formation by blood flow cooling.

We report that ERFA can prolong stent patency and was associated with an improved overall patient survival for unresectable extrahepatic CCA with biliary obstruction. The current study was stronger and more clinically significant than previous studies [13]-[16] [18] [19]. First, there was a larger sample size ( $n=34$ in the ERFA +

Table 4. Multivariate analysis of prognostic factors to predict overall survival after treatment.

\begin{tabular}{cccc}
\hline Factors & Regression coefficient & Standard error & P value \\
\hline Patient age & 0.018 & 0.016 & 0.311 \\
Patient sex & -0.169 & 0.302 & 0.576 \\
Hilar/distal CCA ${ }^{\mathrm{a}}$ & -3.810 & 1.000 & 0.001 \\
Tumor AJCC ${ }^{\mathrm{b}}$ stage & 1.090 & 0.279 & 0.001 \\
Stricture length & -0.088 & 0.148 & 0.555 \\
${\text { Procedure approaches (ERCP } / \text { PTC }^{\mathrm{d}} \text { ) }}_{\text {SEMS }}$ (cover/uncover) & -0.879 & 0.553 & 0.112 \\
Adjuvant chemotherapy & 0.689 & 0.408 & 0.091 \\
Serum albumin level & 0.288 & 0.128 & 0.025 \\
Serum bilirubin level & 0.574 & 0.275 & 0.037 \\
Treatment group & -0.001 & 0.001 & 0.471 \\
\hline
\end{tabular}

${ }^{a}$ Cholangiocarcinoma; ${ }^{b}$ American Joint Committee on Cancer; ${ }^{\mathrm{c}}$ Endoscopic retrograde cholangiopancreatography; ${ }^{\mathrm{d}}$ Percutaneous transhepatic cholangiography; ${ }^{\mathrm{e}} \mathrm{Self}-\mathrm{exp} a n d i n g$ metal stent. 
SEMS group), representing the largest sample for this type of study to our knowledge. The follow-up period was longer than in previous studies. It was used to monitor the long-term outcomes, which showed the actual impact of RFA on both stent patency and overall survival to be lacking. For example, Steel et al. [17] performed endoscopic RF ablation in 6 unresectable CCA patients followed by SEMS placement. However, that study only included a 90-day follow-up regarding biliary patency. Second, stricter selection criteria were used. Here, only patients with unresectable Bismuth type I hilar or distal biliary adenocarcinoma were included. Patients with malignant biliary obstruction caused by other tumors or other Bismuth type of hilar CCAs were excluded, which reduced confounding bias when comparing overall survival rates. Furthermore, we focused on stent patency improvement and survival benefit of ERFA for patients who received SEMS. Patients who received plastic or nasobiliary drainage instead of SEMS shortly after ERFA were excluded which reduced bias when comparing the effectiveness of ERFA because SEMS itself was superior to plastic stent for malignant obstruction jaundice relief [7] [8]. A comprehensive analysis of overall survival was performed. This analysis indicated that when potential prognosis factors (e.g. metastatic disease, covered SEMS) were includedin overall survival analysis, the survival benefit of ERFA for unresectable extrahepatic CCA was confirmed.

Repeat ERFA using a bipolar catheter within the previous metal stent is not always feasible due to technical issues. Based on this study, the metal stent would prevent the bipolar RFA catheter from contacting and ablating the bile duct tumor when performing repeat ERFA [23]. Furthermore, direct contact with the bipolar RFA catheter with the uncovered metal stent could produce a short-circuit, making it impossible for a repeat endobiliary RFA.

In this study, multivariate Cox proportional hazards regression analysis indicated that ERFA, tumor AJCC stage, extrahepatic type, serum albumin and adjuvant chemotherapy were significant prognostic factors for overall patient survival. We attribute this benefit to the fact that ERFA can significantly prolong metal stent patency, which can improve patient comfort by relieving jaundice and chemotherapeutic toxicity both of which contribute to overall survival. As a prognostic factor, survival benefits for bile tract cancer with adjuvant chemotherapy, especially in combination with cisplatin and gemcitabine, is well documented [24]. It is understandable that patients with earlier AJCC extrahepatic CCA or normal serum albumin indicating better hepatic function, had better survival outcomes. We found that distal CCA ostensibly had better outcomes than Bismuth type I hilar CCA patients who received ERFA and/or SEMS but why this occurred is unclear. Nonetheless, even without statistical significance, patients treated with ERCP or those with covered SEMS had better outcomes than those who received the PTC approach $(\mathrm{p}=0.112)$ and those with an uncovered SEMS $(\mathrm{P}=0.091)$, respectively. The PTC approach was used for more advanced stage CCA patients with metastatic disease (6/7, 85.7\%) than those patients treated with the ERCP approach, which may change outcomes. Covered SEMS coated with an organic polymer had longer stent patency than uncovered SEMS likely due to polymer-inhibited tumor ingrowth. Thus, SEMS may also be prognostic although no statistically significant results were observed likely due to the small sample size of patients with covered SEMS (10/76, 13.2\%) were included in this analysis.

The present study has an important weakness: its retrospective nature. Additional clinical studies such as prospective randomized trials are needed to confirm this observation. It is still not possible to claim that endobiliary RFA + SEMS is superior to other methods. However, the present study is meant as a pilot study aiming to investigate the safety and the feasibility of endobiliary RFA.

\section{Conclusion}

Overall, ERFA is safe and effective and it may improve metal stent patency and patient overall survival for EH-CCA with biliary obstruction, and should therefore be incorporated into the treatment algorithm of any center treating patients with EH-CCA.

\section{Disclosures}

No conflict of interest.

\section{Fund}

This work was supported by Pearl River S\&T Nova Program of Guangzhou (2011J2200004) and the grant of Sciences and Technology Committee of Guangdong Province (2011B031800072 and 2013B021800300), China. 


\section{References}

[1] Akamatsu, N., Sugawara, Y. and Hashimoto, D. (2011) Surgical Strategy for Bile Duct Cancer: Advances and Current Limitations. World Journal of Clinical Oncology, 2, 94. http://dx.doi.org/10.5306/wjco.v2.i2.94

[2] Aljiffry, M., Walsh, M.J. and Molinari, M. (2009) Advances in Diagnosis, Treatment and Palliation of Cholangiocarcinoma: 1990-2009. World Journal of Gastroenterology, 15, 4240-4262. http://dx.doi.org/10.3748/wjg.15.4240

[3] Andersen, J.R., Sørensen, S.M., Kruse, A., Rokkjaer, M. and Matzen, P. (1989) Randomised Trial of Endoscopic Endoprosthesis versus Operative Bypass in Malignant Obstructive Jaundice. Gut, 30, 1132-1135. http://dx.doi.org/10.1136/gut.30.8.1132

[4] Goenka, M.K. and Goenka, U. (2014) Palliation: Hilar Cholangiocarcinoma. World Journal of Hepatology, 6, 559-569.

[5] Moss, A.C., Morris, E., Leyden, J. and MacMathuna, P. (2007) Do the Benefits of Metal Stents Justify the Costs? A Systematic Review and Meta-Analysis of Trials Comparing Endoscopic Stents for Malignant Biliary Obstruction. European Journal of Gastroenterology \& Hepatology, 19, 1119-1124. http://dx.doi.org/10.1097/MEG.0b013e3282f16206

[6] Schmassmann, A., von Gunten, E., Knuchel, J., Scheurer, U., Fehr, H.F. and Halter, F. (1996) Wallstents versus Plastic Stents in Malignant Biliary Obstruction: Effects of Stent Patency of the First and Second Stent on Patient Compliance and Survival. The American Journal of Gastroenterology, 91, 654-659.

[7] Yoon, W.J., Ryu, J.K., Yang, K.Y., Paik, W.H., Lee, J.K., Woo, S.M., Park, J.K., Kim, Y.T. and Yoon, Y.B. (2009) A Comparison of Metal and Plastic Stents for the Relief of Jaundice in Unresectable Malignant Biliary Obstruction in Korea: An Emphasis on Cost-Effectiveness in a Country with a low ERCP Cost. Gastrointestinal Endoscopy, 70, 284289. http://dx.doi.org/10.1016/j.gie.2008.12.241

[8] Raju, R.P., Jaganmohan, S.R., Ross, W.A., Davila, M.L., Javle, M., Raju, G.S. and Lee, J.H. (2011) Optimum Palliation of Inoperable Hilar Cholangiocarcinoma: Comparative Assessment of the Efficacy of Plastic and Self-Expanding Metal Stents. Digestive Diseases and Sciences, 56, 1557-1564. http://dx.doi.org/10.1007/s10620-010-1550-5

[9] Loew, B.J., Howell, D.A., Sanders, M.K., Desilets, D.J., Kortan, P.P., May, G.R., Shah, R.J., Chen, Y.K., Parsons, W.G., Hawes, R.H., Cotton, P.B., Slivka, A.A., Ahmad, J., Lehman, G.A., Sherman, S., Neuhaus, H. and Schumacher, B.M. (2009) Comparative Performance of Uncoated, Self-Expanding Metal Biliary Stents of Different Designs in 2 Diameters: Final Results of an International Multicenter, Randomized, Controlled Trial. Gastrointestinal Endoscopy, 70, 445-453. http://dx.doi.org/10.1016/j.gie.2008.11.018

[10] Isayama, H., Komatsu, Y., Tsujino, T., Sasahira, N., Hirano, K., Toda, N., Nakai, Y., Yamamoto, N., Tada, M., Yoshida, H., Shiratori, Y., Kawabe, T. and Omata, M. (2004) A Prospective Randomised Study of “Covered” versus “Uncovered” Diamond Stents for the Management of Distal Malignant Biliary Obstruction. Gut, 53, 729-734. http://dx.doi.org/10.1136/gut.2003.018945

[11] Zoepf, T., Jakobs, R., Arnold, J.C., Apel, D. and Riemann, J.F. (2005) Palliation of Nonresectable Bile Duct Cancer: Improved Survival after Photodynamic Therapy. The American Journal of Gastroenterology, 100, 2426-2430. http://dx.doi.org/10.1111/j.1572-0241.2005.00318.x

[12] Ortner, M.E., Caca, K., Berr, F., Liebetruth, J., Mansmann, U., Huster, D., Voderholzer, W., Schachschal, G., Mössner, J. and Lochs, H. (2003) Successful Photodynamic Therapy for Nonresectable Cholangiocarcinoma: A Randomized Prospective Study. Gastroenterology, 125, 1355-1363. http://dx.doi.org/10.1016/j.gastro.2003.07.015

[13] Tal, A.O., Vermehren, J., Friedrich-Rust, M., Bojunga, J., Sarrazin, C., Zeuzem, S., Trojan, J. and Albert, J.G. (2014) Intraductal Endoscopic Radiofrequency Ablation for the Treatment of Hilar Non-Resectable Malignant Bile Duct Obstruction. World Journal of Gastrointestinal Endoscopy, 6, 13-19. http://dx.doi.org/10.4253/wjge.v6.i1.13

[14] Alis, H., Sengoz, C., Gonenc, M., Kalayci, M.U. and Kocatas, A. (2013) Endobiliary Radiofrequency Ablation for Malignant Biliary Obstruction. Hepatobiliary \& Pancreatic Diseases International, 12, 423-427. http://dx.doi.org/10.1016/S1499-3872(13)60066-1

[15] Strand, D.S., Cosgrove, N.D., Patrie, J.T., Cox, D.G., Bauer, T.W., Adams, R.B., Mann, J.A., Sauer, B.G., Shami, V.M. and Wang, A.Y. (2014) ERCP-Directed Radiofrequency Ablation and Photodynamic Therapy Are Associated with Comparable Survival in the Treatment of Unresectable Cholangiocarcinoma. Gastrointestinal Endoscopy, 80, 794-804. http://dx.doi.org/10.1016/j.gie.2014.02.1030

[16] Rustagi, T. and Jamidar, P.A. (2014) Intraductal Radiofrequency Ablation for Management of Malignant Biliary Obstruction. Digestive Diseases and Sciences, 59, 2635-2641. http://dx.doi.org/10.1007/s10620-014-3237-9

[17] Steel, A.W., Postgate, A.J., Khorsandi, S., Nicholls, J., Jiao, L., Vlavianos, P., Habib, N. and Westaby, D. (2011) Endoscopically Applied Radiofrequency Ablation Appears to Be Safe in the Treatment of Malignant Biliary Obstruction. Gastrointestinal Endoscopy, 73, 149-153. http://dx.doi.org/10.1016/j.gie.2010.09.031 
[18] Wadsworth, C.A., Westaby, D. and Khan, S.A. (2013) Endoscopic Radiofrequency Ablation for Cholangiocarcinoma. Current Opinion in Gastroenterology, 29, 305-311. http://dx.doi.org/10.1097/MOG.0b013e32835faacc

[19] Figueroa-Barojas, P., Bakhru, M.R., Habib, N.A., Ellen, K., Millman, J., Jamal-Kabani, A., Gaidhane, M. and Kahaleh, M. (2013) Safety and Efficacy of Radiofrequency Ablation in the Management of Unresectable Bile Duct and Pancreatic Cancer: A Novel Palliation Technique. Journal of Oncology, 2013, 1-5. http://dx.doi.org/10.1155/2013/910897

[20] National Comprehensive Cancer Network (2010) NCCN Clinical Practice Guidelines in Oncology: Hepatiobiliary Cancers, V.1.2010. http://www.nccn.org/professionals/physician_gls/PDF/hepatobiliary.pdf

[21] Dindo, D., Demartines, N. and Clavien, P.A. (2004) Classification of Surgical Complications: A New Proposal with Evaluation in a Cohort of 6336 Patients and Results of a Survey. Annals of Surgery, 240, 205-213. http://dx.doi.org/10.1097/01.sla.0000133083.54934.ae

[22] Goldberg, S.N., Hahn, P.F., Tanabe, K.K., Mueller, P.R., Schima, W., Athanasoulis, C.A., Compton, C.C., Solbiati, L. and Gazelle, G.S. (1998) Percutaneous Radiofrequency Tissue Ablation: Does Perfusion-Mediated Tissue Cooling Limit Coagulation Necrosis? Journal of Vascular and Interventional Radiology, 9, 101-111. http://dx.doi.org/10.1016/S1051-0443(98)70491-9

[23] Pai, M., Valek, V., Tomas, A., Doros, A., Quaretti, P., Golfieri, R., Mosconi, C. and Habib, N. (2014) Percutaneous Intraductal Radiofrequency Ablation for Clearance of Occluded Metal Stent in Malignant Biliary Obstruction: Feasibility and Early Results. CardioVascular and Interventional Radiology, 37, 235-240. http://dx.doi.org/10.1007/s00270-013-0688-x

[24] Valle, J., Wasan, H., Palmer, D.H., Cunningham, D., Anthoney, A., Maraveyas, A., Madhusudan, S., Iveson, T., Hughes, S., Pereira, S.P., Roughto, M. and Bridgewater, J. (2010) Cisplatin plus Gemcitabine versus Gemcitabine for Biliary Tract Cancer. New England Journal of Medicine, 362, 1273-1281. http://dx.doi.org/10.1056/NEJMoa0908721 